\title{
Using Annual Panel Data To Examine The Monday Effect
}

Matthew R. Morey, Pace University, USA

Menahem Rosenberg, Touro College, USA

\begin{abstract}
The Monday effect is a well-known anomaly in which Monday stocks returns are significantly different from other days. Recent research suggests that small-cap stocks exhibit negative and significant Monday returns, mid-cap stocks show no Monday effect and large-cap stocks have positive and significant Monday returns. In this short paper we re-examine the Monday effect using a somewhat different approach that the rest of the literature. Specifically, we examine the U.S. mean Monday returns for each market capitalization decile and for each year over the period 1966-2007. We then examine the patterns of these annual Monday returns. Using this method, we find that the Monday effect has dissipated for all sizes of stocks so much that, by the middle 1990s, the Monday returns are generally not significant from zero.
\end{abstract}

Keywords: Monday Effect; Dissipation

\section{INTRODUCTION}

C

$\mathrm{n}$ the 1980's a number of studies (French (1980), Keim and Stambaugh (1983) and Lakonishok and Smidt (1988)) reported the existence of a Monday effect, where Monday returns were significantly negative in the U.S. stock market. ${ }^{1}$ However, more recent research, using more current data, suggests that the Monday effect depends on the size of the firm. In a review of the extensive Monday effect literature, Pettengill (2003a) reports that small-cap stocks have negative and significant Monday returns, mid-cap stocks show no Monday effect and large-cap stocks have positive and significant Monday returns. ${ }^{2}$

In this paper we re-examine the relationship between firm size and the Monday effect using a somewhat different approach than others have used in the literature. Specifically we use an annual panel approach where we examine the Monday effect for each market capitalization decile and for each year over the period 1966-2007 and then examine the patterns of these annual Monday tests. Using this method, we find that the Monday effect has dissipated for all sizes of stocks so much, that by the middle 1990s, the Monday returns are generally not significant from zero.

The rest of this short paper is organized as follows. In Section 2 we describe out data. In Section 3 we present our model for estimating the Monday effect. In Section 4 we present our results and we conclude in Section 5.

\footnotetext{
${ }^{1}$ Along with the existence of the Monday effect, there is a long literature that has tried to explain the Monday effect. The explanations put forward include individual investors selling more on Friday than they buy on Monday (Lakonishok and Maberly (1990) and Dyl and Maberly (1992)); negative information disclosure on Fridays (which pushes down Monday returns) (Penman (1987) and Damodaran (1989)); short-selling speculators closing out their positions on Friday and re-opening on Monday (the reopening causes prices to fall on Monday) (Chen and Singal (2003); and transactions costs being too high to arbitrage away the effect (Kamara (1997).

${ }^{2}$ More specifically, Kamara (1997) finds that the Monday effect holds for small capitalization stocks. Conversely, Brusa, Pu, and Schulman (2000, 2003a, 2003b and 2005) and Gu (2004) and Pettengill (2003b) show that while the Monday effect does not hold for mid-cap stocks there exists a 'reverse' Monday effect in large firms, where Monday returns, instead of being significantly negative, are significantly positive.
}

(C) 2012 The Clute Institute http://www.cluteinstitute.com/ 


\section{DATA}

Since we examine the relationship between the Monday effect and firm size, we use the daily NYSEAMEX and NASDAQ indices that are based on capitalization size. Specifically there are ten different capitalization deciles with 1 being the smallest stocks and 10 being the largest stocks. We gather data over the period from January 1966 through December 2007 for the NYSE-AMEX and from January 1973 through December 2007 for the NASDAQ. Hence, we have 42 annual observations for NYSE-AMEX and 35 annual observations for NASDAQ.

\section{MODEL}

To measure the Monday effect we use equation (1)

$r_{t}=\mu+\beta D_{t}+\varepsilon_{t}$

where $r_{t}$ is the daily return and the $D_{t}$ is dummy for Mondays.

For the residuals in equation (1) it is well-known since Engle (1982) that stocks returns do not display constant variance. To deal with the non-constant variance, we use EGARCH as opposed to GARCH as EGARCH provided higher log likelihood than GARCH in the majority of the regressions using equation (1). Moreover, Kim and Kun (1994) have found that E-GARCH provides a better fit for index returns, which is what we essentially use in this paper. Note however, that the results using GARCH are very similar to those presented in the paper. We do not report the GARCH results but they are available upon request.

\section{RESULTS}

In Table 1 we examine the trend in the Monday effect over the period 1966-2007. To do this we first separate stocks into ten deciles by capitalization size with the first decile being the smallest capitalization stocks and the tenth being the largest capitalization stocks. Then for each decile of capitalization, we calculate the annual mean Monday returns for the period 1966-2007 for NYSE-AMEX stocks, and for 1973-2007 for the NASDAQ stocks. Hence, for each capitalization decile we have 42 annual mean Monday returns for the NYSE-AMEX stocks and 35 annual mean Monday returns for the NASDAQ stocks. Then, for each year of data and for each decile of market capitalization, we conduct a Wald Test to examine the strength of the Monday effect. The Wald Test examines the null hypothesis that annual mean Monday returns are zero. Hence, a rejection of the null indicates the existence of a Monday effect, whether negative or positive returns. As the Monday effect lessens (increases) the Wald test statistic should diminish (gain) in size.

Finally, for each capitalization decile, we examine the Spearman-rank correlation test between the Wald statistic and the year of those mean annual Monday returns. We define year as increasing as the year gets closer to the present. Hence, using the NYSE/AMEX stocks, 1966 is 1 and 2007 is 42 . For the NASDAQ stocks, 1973 is 1 and 2007 is 35. If the Spearman rank correlation is negative and significant it indicates that as the years get closer to the present the Wald test statistics are getting smaller. This would indicate that the Monday effect is getting smaller as we get closer to 2007. If the Spearman rank correlation is positive and significant it indicates that as the years get closer to the present the Wald test statistics are getting larger. This would indicate the Monday effect is getting larger as we get closer to 2007.

Table 1 shows a that there is a clear dissipation of the Monday effect in all sizes of stock as the rank correlation is negative for each decile. Moreover, the negative rank correlation is significant at traditional levels for all but the smallest cap deciles. Hence, as time proceeds from 1966, the Wald test statistics are generally becoming smaller and thus indicative of a diminishing Monday effect.

In Tables 2-3 we report the significance of the Wald Tests used in Table 1. We test whether the Wald Statistics for the annual Monday return (for each decile) are significant from zero at the 5\% level of significance. To make these tables more readable, instead of reporting the actual level of the Wald statistic we instead report "False" 
if the Wald statistic is not significant from zero at the $5 \%$ level. Similarly we report "****" if the Wald statistic is significantly different from zero at the $5 \%$ level. In Table 2 we provide the result using the NYSE-AMEX indices and Table 3 provides results using the NASDAQ indices.

Tables 2-3 show that over time the returns on Monday become insignificant from zero for all firm size levels, i.e., small cap, mid cap or large cap. Indeed, for the small-cap stocks we find that the Monday effect is not significant for the vast majority of the years after the early 1990s (note that even before the 1990s there is a large number of cases where the Wald statistic for the smallest cap decile is not significant). For the NASDAQ stocks, we see the same type of results after the period 1999. Hence, unlike what has been reported recently in the literature, we find relatively little evidence of a negative and significant Monday effect in small caps.

Also distinct from recent reports in the literature, we find relatively little evidence for a positive and significant Monday effect in large-cap stocks. For both NYSE-AMEX and NASDAQ we find that large-cap stocks do not show a significant Monday effect after the early 1990s. Indeed, in only one case since 1993 are the Monday returns significantly different from zero in the tenth decile using the NYSE-AMEX or NASDAQ.

As a robustness check for our results in Tables 2-3 we perform the same test, but instead of using the standard five percent significance threshold we use the more generous $10 \%$ significance level. In this way we make it easier for the Monday effect to be significant. The results are presented in Tables 4 and 5 for the NYSE-AMEX and NASDAQ, respectively. The results are fairly similar to those using the 5\% significance threshold. The major difference in the results between the $10 \%$ and $5 \%$ results is that in the smallest capitalization stocks we see more significant Monday returns for the middle 1990s and the period 2005-2007. Other than this the results are generally the same.

\section{CONCLUSIONS}

The Monday effect is a very well-known anomaly in which Monday returns are significantly different from zero. Since early 1980s there have been a slew of papers that have examined this issue. Most recent papers find that the Monday effect has changed since first being observed. Indeed, most recent research seems to indicate that there is negative return Monday effect in small-cap stocks, no Monday effect in mid-cap stocks and a positive Monday effect in big-cap stocks.

In this paper we re-examine the well-known Monday effect using a different type of approach than is typically found in the literature. Specifically we examine the Monday effect for each market capitalization decile and for each year over the period 1966-2007. We then examine the patterns of these annual Monday returns tests. Using these data, we find that the Monday effect has dissipated for all firm sizes of stocks so much that by middle 1990s the Monday returns are generally not significant from zero at the five percent using NYSE-AMEX. We find with the NASDAQ indices that the dissipation takes a little longer but still is there. Indeed, by the later 1990s the Monday returns for the NASDAQ deciles are generally not significant using the 5\% level of significance. Hence, unlike recent research, we do not much evidence of a positive and significant Monday effect in big-cap stocks after the mid 1990s nor do we find much evidence of small-cap stocks exhibiting a significant negative Monday effect after the late 1990s. Instead we find that in terms of statistical significance the Monday effect has dissipated over the years to such an existent that it does not seem to hold much.

Our paper is not without its caveats. Recent research by Doyle and Chan (2009) has indicated that there is wandering Monday effect that comes and goes. Consequently, it may be that our findings of a dissipation of the Monday effect across all firm sizes is a transitory one and that the Monday effect will spring up again. It may also be that a Monday effect holds at lower significance levels, which means the effect is still there but not statistically significant at traditional levels. Furthermore, some recent research (Wang and Erickson (1997) and Sun and Tong (2002)) has found that the Monday effect only holds in certain periods such as the end of the month or during nonbear markets. We have not investigated these issues directly as they beyond the scope of this paper but they may indeed hold. 
Finally it may also be that when years are aggregated together in large samples (as opposed to examining the years individually) the results change. Indeed, it is easier to find significance when using large samples. However, if the Monday effect really holds the results should be robust to different sample periods and sizes. Our study has documented that when the Monday effect is examined using a annual panel approach the annual Monday returns are not significantly different from zero after middle 1990s for NYSE-AMEX and after the later 1990s for NASDAQ. Hence, our results cast doubt on the Monday effect holding in the recent past.

\section{AUTHOR INFORMATION}

Matthew R. Morey, Department of Finance, Lubin School of Business, Pace University, E-mail: mmorey@pace.edu

Menahem Rosenberg, Department of Business, Touro College, USA. E-mail: MenahemR@Touro.edu. Corresponding author.

\section{REFERENCES}

1. Boudreaux, Denis, Spurma Rao, and Phillip Fuller, 2010, “An investigation of the weekend effect during different market orientations", Journal of Economics and Finance, 34, 257-268.

2. Brusa, J., L. Pu, and C. Schulman, "The Monday Effect, 'Reverse' Monday Effect, and Firm Size," Journal of Business Finance and Accounting (June/July 2000), pp. 555-574.

3. Brusa J, L. Pu., C. Schulman, 2003a, "The weekend and 'reverse' weekend effects: an analysis by month of the year, week of the month, and industry", Journal of Business Finance and Accounting, 30, 5/6, 863890.

4. Brusa J, L. Pu, C. Schulman, 2003b, "The "reverse" weekend effect: the U.S. market versus international markets" International Review of Financial Analysis, 12, 3, 267.

5. Brusa J, L. Pu, C. Schulman, 2005, "Weekend effect, 'reverse' weekend effect, and investor trading activities", Journal of Business Accounting, 32, 7/8, 1495.

6. Connolly, Robert A. 1989. An Examination of the Robustness of the Monday Effect. Journal of Financial and Quantitative Analysis 24(2):133-69.

7. Doyle, J.R., Chen, C.H., 2009. The wandering weekday effect in major stock markets. Journal of Banking and Finance 33, 1388-1399.

8. Engle, Robert F. (1982). "Autoregressive Conditional Heteroscedasticity with Estimates of Variance of United Kingdom Inflation", Econometrica 50:987-1008.

9. $\quad$ French, Kenneth R. 1980. Stock Returns and the Monday Effect. Journal of Financial Economics 8(1):5569.

10. French, Kenneth R., William G. Schwert, and Robert F. Stambaugh. 1987, Expected Stock Returns and Volatility. Journal of Financial Economics 19, 3-29.

11. Gu, A., 2004, "The reversing weekend effect: evidence from the U.S. equity markets", Review of Quantitative Finance and Accounting, 22,1, 5

12. Kamara, Avraham, 1997, New Evidence on the Monday Seasonal in Stock Returns, Journal of Business, 70(1), 63-84.

13. Kim D. and S. Kon, "Alternative Models for the Conditional Heteroscedasticity of Stock Returns", Journal of Business, 1994, 67(4), pp 563-598

14. Keim, D.B. and Stambaugh R.F., 1984, A Further Investigation of the Monday Effect, Journal of Finance, 39:819-835.

15. Lakonishok, Josef, and Seymour Smidt. 1988, Are Seasonal Anomalies Real? A Ninety-Year Perspective. Review of Financial Studies 1(4):403-25.

16. Lakonishok, Josef, and Edwin Maberly, 1990, The Monday Effect: Trading Patterns of Individual and Institutional Investors, The Journal of Finance, 45(1), pp 231-243.

17. Penman, Stephen. H. (1987), The Distribution of earning news over time and seasonalities in aggregate stock returns, Journal of Financial Economics, 18, 199-228.

18. Pettengill, Glenn, N., 2003, “A Survey of the Monday Effect Literature”, Quarterly Journal of Business and Economics, 42, 3-27. 
19. Pettengill, G., J. Wingender, and R. Kohli, "Arbitrage, Institutional Investors, and the Monday Effect," Quarterly Journal of Business and Economics, 42, 49-65.

20. Schwert, William, G., 2003 Anomalies and Market Efficiency, in Handbook of Economics of Finance, Chapter 15, G. M. Constantinides and R Stultz (Eds), Elsevier Science, Amsterdam.

21. Sun Qian and Wilson H.S. Tong, 2002, Another New Look at the Monday Effect, Journal of Business Finance and Accounting, 29 (7) \& (8): 1123-1147.

22. Wang, Ko, Yuming Li, and John Erickson. 1997. A New Look at Monday Effect. Journal of Finance 52(5):2171-86. 


\section{APPENDIX}

Table 1: Spearman-Rank Correlation Test Results Between Annual Wald Test (as a Measure of Monday effect strength) and Year (where the year is increasing as we move to the present) by Capitalization Decile

\begin{tabular}{|c|c|c|}
\hline $\begin{array}{c}\text { Capitalization Decile (Lowest to } \\
\text { Highest) }\end{array}$ & NYSE-AMEX Stocks & $\begin{array}{l}\text { NASDAQ } \\
\text { Stocks }\end{array}$ \\
\hline \multirow{2}{*}{1} & -.1835 & -.2500 \\
\hline & $(.2446)$ & $(.1477)$ \\
\hline \multirow{2}{*}{2} & -.2082 & $-.2940 *$ \\
\hline & $(.1859)$ & $(.0867)$ \\
\hline \multirow{2}{*}{3} & $-.3106^{* *}$ & $-.3650 * *$ \\
\hline & $(.0453)$ & $(.0311)$ \\
\hline \multirow{2}{*}{4} & $-.4284 * *$ & $-.3180^{*}$ \\
\hline & $(.0046)$ & $(.0630)$ \\
\hline \multirow{2}{*}{5} & $-.4304 * * *$ & $-.2860 *$ \\
\hline & $(.0044)$ & $(.0958)$ \\
\hline \multirow{2}{*}{6} & $-.3684 * *$ & $-.5950 * * *$ \\
\hline & $(.0164)$ & $(.0002)$ \\
\hline \multirow{2}{*}{7} & $-.4667 * * *$ & $-.6230 * * *$ \\
\hline & $(.0018)$ & $(.0001)$ \\
\hline \multirow{2}{*}{8} & $-.4211 * * *$ & $-.5250 * * *$ \\
\hline & $(.0055)$ & $(.0012)$ \\
\hline \multirow{2}{*}{9} & $-.5307 * * *$ & $-.6250 * * *$ \\
\hline & $(.0003)$ & $(.0001)$ \\
\hline \multirow{2}{*}{10} & $-.4628 * * *$ & $-.5570 * * *$ \\
\hline & $(.0020)$ & $(.0005)$ \\
\hline
\end{tabular}

In this table we first separate stocks into 10 deciles by capitalization size with decile 1 being the smallest capitalization stocks and decile 10 being the largest capitalization stocks. Then for each decile of capitalization, we calculate the annual mean Monday excess returns for the period 1966-2007 for NYSE-AMEX stocks and for the period 1973-2007 for the NASDAQ stocks. Hence, for each capitalization decile we have 42 annual excess returns for the NYSE-AMEX stocks and 35 annual excess returns for the NASDAQ stocks. Then, for each decile for each year of data we conduct a Wald Test to examine the strength of the Monday effect for that decile during that year. As the Monday effect lessens (increases) the Wald test statistic should be smaller (larger). Next, for each capitalization decile, we examine the Spearman-rank correlation test between the Wald statistic and the year of those excess Monday returns. We define year as increasing as the year gets closer to the present. Hence, using the NYSE/AMEX stocks, 1966 is 1 and 2007 is 42 . For the NASDAQ stocks, 1973 is 1 and 2007 is 35 . If the Spearman rank correlation is negative and significant it indicates that as the years get closer to the present the annual Wald tests are getting smaller. This would indicate that the Monday effect is getting smaller as we get closer to the present period. Note that the p-values are in parenthesis and $* * *$, **, * indicate that the rank correlation between the annual Wald tests and increasing years is significant at the one, five and ten percent levels respectively. 
Table 2: Wald Tests Results Annual Excess Mean Monday Returns by Year and Capitalization Decile for NYSE-AMEX Stocks

\begin{tabular}{|c|c|c|c|c|c|c|c|c|c|c|}
\hline Year & Decile 1 & Decile 2 & Decile 3 & Decile 4 & Decile 5 & Decile 6 & Decile 7 & Decile 8 & Decile 9 & Decile10 \\
\hline 1966 & FALSE & $* * *$ & FALSE & FALSE & FALSE & FALSE & FALSE & FALSE & FALSE & FALSE \\
\hline 1967 & $* * *$ & $* * *$ & $* * *$ & $* * *$ & FALSE & $* * *$ & $* * *$ & $* * *$ & $* * *$ & $* * *$ \\
\hline 1968 & FALSE & FALSE & FALSE & FALSE & FALSE & FALSE & FALSE & FALSE & FALSE & FALSE \\
\hline 1969 & $* * *$ & $* * *$ & $* * *$ & $* * *$ & $* * *$ & $* * *$ & $* * *$ & $* * *$ & $* * *$ & $* * *$ \\
\hline 1970 & FALSE & FALSE & FALSE & $* * *$ & $* * *$ & $* * *$ & $* * *$ & FALSE & $* * *$ & $* * *$ \\
\hline 1971 & $* * *$ & FALSE & FALSE & FALSE & FALSE & FALSE & FALSE & FALSE & FALSE & FALSE \\
\hline 1972 & FALSE & FALSE & $* * *$ & $* * *$ & $* * *$ & $* * *$ & $* * *$ & $* * *$ & $* * *$ & $* * *$ \\
\hline 1973 & $* * *$ & $* * *$ & $* * *$ & $* * *$ & $* * *$ & $* * *$ & $* * *$ & $* * *$ & $* * *$ & $* * *$ \\
\hline 1974 & $* * *$ & $* * *$ & $* * *$ & $* * *$ & $* * *$ & $* * *$ & FALSE & $* * *$ & FALSE & FALSE \\
\hline 1975 & FALSE & FALSE & FALSE & FALSE & FALSE & FALSE & FALSE & FALSE & FALSE & FALSE \\
\hline 1976 & FALSE & $* * *$ & FALSE & FALSE & FALSE & FALSE & FALSE & FALSE & FALSE & FALSE \\
\hline 1977 & FALSE & FALSE & FALSE & $* * *$ & $* * *$ & FALSE & FALSE & FALSE & FALSE & FALSE \\
\hline 1978 & $* * *$ & FALSE & $* * *$ & $* * *$ & $* * *$ & $* * *$ & $* * *$ & $* * *$ & FALSE & FALSE \\
\hline 1979 & FALSE & FALSE & $* * *$ & FALSE & $* * *$ & $* * *$ & $* * *$ & $* * *$ & FALSE & FALSE \\
\hline 1980 & $* * *$ & FALSE & FALSE & $* * *$ & $* * *$ & $* * *$ & $* * *$ & $* * *$ & $* * *$ & FALSE \\
\hline 1981 & $* * *$ & $* * *$ & $* * *$ & $* * *$ & $* * *$ & $* * *$ & $* * *$ & $* * *$ & $* * *$ & FALSE \\
\hline 1982 & FALSE & $* * *$ & $* * *$ & *** & $* * *$ & FALSE & $* * *$ & FALSE & FALSE & FALSE \\
\hline 1983 & FALSE & FALSE & FALSE & FALSE & $* * *$ & FALSE & FALSE & $* * *$ & FALSE & FALSE \\
\hline 1984 & $* * *$ & $* * *$ & $* * *$ & $* * *$ & $* * *$ & $* * *$ & $* * *$ & $* * *$ & $* * *$ & FALSE \\
\hline 1985 & $* * *$ & FALSE & $* * *$ & $* * *$ & $* * *$ & $* * *$ & FALSE & FALSE & FALSE & FALSE \\
\hline 1986 & $* * *$ & $* * *$ & $* * *$ & $* * *$ & $* * *$ & $* * *$ & $* * *$ & $* * *$ & $* * *$ & $* * *$ \\
\hline 1987 & $* * *$ & $* * *$ & $* * *$ & $* * *$ & $* * *$ & $* * *$ & $* * *$ & $* * *$ & $* * *$ & FALSE \\
\hline 1988 & FALSE & FALSE & $* * *$ & FALSE & FALSE & $* * *$ & $* * *$ & FALSE & FALSE & FALSE \\
\hline 1989 & FALSE & $* * *$ & $* * *$ & *** & $* * *$ & $* * *$ & $* * *$ & $* * *$ & FALSE & FALSE \\
\hline 1990 & $* * *$ & $* * *$ & $* * *$ & $* * *$ & $* * *$ & FALSE & FALSE & FALSE & FALSE & FALSE \\
\hline 1991 & $* * *$ & $* * *$ & $* * *$ & $* * *$ & FALSE & $* * *$ & FALSE & $* * *$ & FALSE & FALSE \\
\hline 1992 & FALSE & FALSE & FALSE & FALSE & FALSE & FALSE & FALSE & FALSE & FALSE & $* * *$ \\
\hline 1993 & FALSE & $* * *$ & FALSE & FALSE & FALSE & FALSE & FALSE & FALSE & FALSE & FALSE \\
\hline 1994 & FALSE & FALSE & FALSE & FALSE & FALSE & FALSE & FALSE & FALSE & FALSE & FALSE \\
\hline 1995 & $* * *$ & $* * *$ & FALSE & $* * *$ & FALSE & $* * *$ & FALSE & FALSE & FALSE & FALSE \\
\hline 1996 & FALSE & FALSE & $* * *$ & FALSE & FALSE & FALSE & FALSE & FALSE & FALSE & FALSE \\
\hline 1997 & FALSE & FALSE & FALSE & FALSE & FALSE & FALSE & FALSE & FALSE & FALSE & FALSE \\
\hline 1998 & $* * *$ & $* * *$ & $* * *$ & FALSE & FALSE & FALSE & FALSE & FALSE & FALSE & FALSE \\
\hline 1999 & FALSE & FALSE & FALSE & FALSE & FALSE & FALSE & FALSE & FALSE & FALSE & FALSE \\
\hline 2000 & FALSE & FALSE & FALSE & FALSE & $* * *$ & FALSE & $* * *$ & $* * *$ & FALSE & $* * *$ \\
\hline 2001 & FALSE & FALSE & $* * *$ & FALSE & FALSE & $* * *$ & FALSE & FALSE & FALSE & FALSE \\
\hline 2002 & FALSE & FALSE & FALSE & $* * *$ & FALSE & FALSE & FALSE & FALSE & FALSE & FALSE \\
\hline 2003 & FALSE & FALSE & FALSE & FALSE & FALSE & FALSE & FALSE & FALSE & FALSE & FALSE \\
\hline 2004 & FALSE & $* * *$ & FALSE & FALSE & FALSE & FALSE & FALSE & FALSE & FALSE & FALSE \\
\hline 2005 & $* * *$ & FALSE & FALSE & FALSE & FALSE & FALSE & FALSE & FALSE & FALSE & FALSE \\
\hline 2006 & FALSE & $* * *$ & FALSE & FALSE & FALSE & FALSE & FALSE & FALSE & FALSE & FALSE \\
\hline 2007 & FALSE & FALSE & FALSE & FALSE & FALSE & FALSE & FALSE & FALSE & FALSE & FALSE \\
\hline
\end{tabular}

(Note: "False" indicates that the Monday returns are not significantly different from zero using the five percent level of significance, $* * *$ indicates the Monday returns are significantly different from zero at the five percent level ) 
Table 3: Wald Tests Results Annual Excess Mean Monday Returns by Year and Capitalization Decile for NASDAQ Stocks

\begin{tabular}{|c|c|c|c|c|c|c|c|c|c|c|}
\hline Year & Decile 1 & Decile 2 & Decile 3 & Decile 4 & Decile 5 & Decile 6 & Decile 7 & Decile 8 & Decile 9 & Decile10 \\
\hline 1973 & $* * *$ & $* * *$ & $* * *$ & $* * *$ & $* * *$ & $* * *$ & $* * *$ & $* * *$ & $* * *$ & $* * *$ \\
\hline 1974 & $* * *$ & $* * *$ & $* * *$ & $* * *$ & FALSE & $* * *$ & $* * *$ & $* * *$ & $* * *$ & FALSE \\
\hline 1975 & FALSE & FALSE & FALSE & FALSE & FALSE & FALSE & FALSE & FALSE & FALSE & FALSE \\
\hline 1976 & $* * *$ & $* * *$ & $* * *$ & $* * *$ & $* * *$ & FALSE & $* * *$ & FALSE & FALSE & FALSE \\
\hline 1977 & $* * *$ & FALSE & FALSE & FALSE & $* * *$ & $* * *$ & $* * *$ & FALSE & $* * *$ & FALSE \\
\hline 1978 & $* * *$ & FALSE & $* * *$ & $* * *$ & $* * *$ & $* * *$ & $* * *$ & $* * *$ & $* * *$ & $* * *$ \\
\hline 1979 & FALSE & $* * *$ & FALSE & FALSE & $* * *$ & FALSE & $* * *$ & FALSE & $* * *$ & $* * *$ \\
\hline 1980 & $* * *$ & $* * *$ & $* * *$ & $* * *$ & FALSE & $* * *$ & $* * *$ & $* * *$ & $* * *$ & $* * *$ \\
\hline 1981 & $* * *$ & $* * *$ & $* * *$ & $* * *$ & $* * *$ & $* * *$ & $* * *$ & $* * *$ & $* * *$ & $* * *$ \\
\hline 1982 & $* * *$ & $* * *$ & $* * *$ & $* * *$ & $* * *$ & $* * *$ & $* * *$ & $* * *$ & $* * *$ & FALSE \\
\hline 1983 & FALSE & $* * *$ & $* * *$ & $* * *$ & $* * *$ & $* * *$ & $* * *$ & $* * *$ & $* * *$ & $* * *$ \\
\hline 1984 & $* * *$ & $* * *$ & $* * *$ & $* * *$ & $* * *$ & $* * *$ & $* * *$ & $* * *$ & $* * *$ & $* * *$ \\
\hline 1985 & FALSE & $* * *$ & $* * *$ & $* * *$ & $* * *$ & $* * *$ & $* * *$ & $* * *$ & $* * *$ & $* * *$ \\
\hline 1986 & $* * *$ & $* * *$ & $* * *$ & $* * *$ & $* * *$ & $* * *$ & $* * *$ & $* * *$ & $* * *$ & $* * *$ \\
\hline 1987 & $* * *$ & $* * *$ & $* * *$ & $* * *$ & $* * *$ & $* * *$ & $* * *$ & $* * *$ & $* * *$ & $* * *$ \\
\hline 1988 & FALSE & $* * *$ & $* * *$ & $* * *$ & $* * *$ & FALSE & $* * *$ & $* * *$ & $* * *$ & FALSE \\
\hline 1989 & $* * *$ & $* * *$ & $* * *$ & $* * *$ & $* * *$ & $* * *$ & $* * *$ & $* * *$ & $* * *$ & $* * *$ \\
\hline 1990 & FALSE & $* * *$ & $* * *$ & $* * *$ & $* * *$ & $* * *$ & $* * *$ & $* * *$ & FALSE & FALSE \\
\hline 1991 & FALSE & $* * *$ & $* * *$ & $* * *$ & $* * *$ & $* * *$ & $* * *$ & $* * *$ & $* * *$ & FALSE \\
\hline 1992 & FALSE & $* * *$ & $* * *$ & $* * *$ & $* * *$ & $* * *$ & FALSE & FALSE & FALSE & FALSE \\
\hline 1993 & FALSE & $* * *$ & $* * *$ & $* * *$ & $* * *$ & $* * *$ & $* * *$ & $* * *$ & FALSE & FALSE \\
\hline 1994 & $* * *$ & $* * *$ & $* * *$ & $* * *$ & FALSE & FALSE & FALSE & FALSE & FALSE & FALSE \\
\hline 1995 & $* * *$ & FALSE & $* * *$ & $* * *$ & $* * *$ & $* * *$ & FALSE & FALSE & FALSE & FALSE \\
\hline 1996 & $* * *$ & FALSE & $* * *$ & FALSE & FALSE & FALSE & FALSE & FALSE & FALSE & FALSE \\
\hline 1997 & $* * *$ & $* * *$ & $* * *$ & FALSE & FALSE & FALSE & FALSE & FALSE & FALSE & FALSE \\
\hline 1998 & $* * *$ & $* * *$ & $* * *$ & $* * *$ & $* * *$ & FALSE & $* * *$ & FALSE & FALSE & FALSE \\
\hline 1999 & FALSE & FALSE & FALSE & FALSE & FALSE & FALSE & FALSE & FALSE & FALSE & FALSE \\
\hline 2000 & FALSE & $* * *$ & FALSE & FALSE & FALSE & FALSE & FALSE & FALSE & FALSE & FALSE \\
\hline 2001 & $* * *$ & FALSE & FALSE & FALSE & FALSE & FALSE & FALSE & FALSE & FALSE & FALSE \\
\hline 2002 & $* * *$ & $* * *$ & $* * *$ & FALSE & $* * *$ & FALSE & FALSE & FALSE & FALSE & FALSE \\
\hline 2003 & FALSE & FALSE & FALSE & FALSE & FALSE & FALSE & FALSE & FALSE & FALSE & FALSE \\
\hline 2004 & FALSE & FALSE & $* * *$ & FALSE & FALSE & FALSE & FALSE & FALSE & FALSE & FALSE \\
\hline 2005 & FALSE & FALSE & FALSE & FALSE & FALSE & FALSE & FALSE & FALSE & FALSE & FALSE \\
\hline 2006 & FALSE & FALSE & FALSE & $* * *$ & FALSE & FALSE & FALSE & FALSE & FALSE & FALSE \\
\hline 2007 & FALSE & FALSE & FALSE & FALSE & FALSE & FALSE & FALSE & FALSE & FALSE & FALSE \\
\hline
\end{tabular}

(Note: "False" indicates that the Monday returns are not significantly different from zero using the five percent level of significance, $* * *$ indicates the Monday returns are significantly different from zero at the five percent level ) 
Table 4: Wald Tests Results Annual Excess Mean Monday Returns by Year and Capitalization Decile for NYSE-AMEX Stocks

\begin{tabular}{|c|c|c|c|c|c|c|c|c|c|c|}
\hline Year & Decile 1 & Decile 2 & Decile 3 & Decile 4 & Decile 5 & Decile 6 & Decile 7 & Decile 8 & Decile 9 & Decile10 \\
\hline 1966 & FALSE & $* * *$ & FALSE & FALSE & FALSE & FALSE & FALSE & FALSE & FALSE & FALSE \\
\hline 1967 & $* * *$ & $* * *$ & $* * *$ & $* * *$ & $* * *$ & $* * *$ & $* * *$ & $* * *$ & $* * *$ & $* * *$ \\
\hline 1968 & FALSE & FALSE & FALSE & FALSE & FALSE & FALSE & FALSE & FALSE & FALSE & FALSE \\
\hline 1969 & $* * *$ & $* * *$ & $* * *$ & $* * *$ & $* * *$ & $* * *$ & $* * *$ & $* * *$ & $* * *$ & $* * *$ \\
\hline 1970 & $* * *$ & $* * *$ & $* * *$ & $* * *$ & $* * *$ & $* * *$ & $* * *$ & $* * *$ & $* * *$ & $* * *$ \\
\hline 1971 & $* * *$ & FALSE & FALSE & FALSE & FALSE & FALSE & FALSE & FALSE & FALSE & FALSE \\
\hline 1972 & FALSE & FALSE & $* * *$ & $* * *$ & $* * *$ & $* * *$ & $* * *$ & $* * *$ & $* * *$ & $* * *$ \\
\hline 1973 & $* * *$ & $* * *$ & $* * *$ & $* * *$ & $* * *$ & $* * *$ & $* * *$ & $* * *$ & $* * *$ & $* * *$ \\
\hline 1974 & $* * *$ & $* * *$ & $* * *$ & $* * *$ & $* * *$ & $* * *$ & $* * *$ & $* * *$ & FALSE & FALSE \\
\hline 1975 & FALSE & FALSE & FALSE & $* * *$ & FALSE & FALSE & FALSE & FALSE & FALSE & FALSE \\
\hline 1976 & FALSE & $* * *$ & FALSE & FALSE & FALSE & $* * *$ & FALSE & FALSE & FALSE & FALSE \\
\hline 1977 & FALSE & FALSE & FALSE & $* * *$ & $* * *$ & $* * *$ & $* * *$ & FALSE & $* * *$ & FALSE \\
\hline 1978 & $* * *$ & $* * *$ & $* * *$ & $* * *$ & $* * *$ & $* * *$ & $* * *$ & $* * *$ & $* * *$ & FALSE \\
\hline 1979 & FALSE & FALSE & $* * *$ & $* * *$ & $* * *$ & $* * *$ & $* * *$ & $* * *$ & $* * *$ & FALSE \\
\hline 1980 & $* * *$ & FALSE & FALSE & $* * *$ & $* * *$ & $* * *$ & $* * *$ & $* * *$ & $* * *$ & FALSE \\
\hline 1981 & $* * *$ & $* * *$ & $* * *$ & $* * *$ & $* * *$ & $* * *$ & $* * *$ & $* * *$ & $* * *$ & FALSE \\
\hline 1982 & FALSE & $* * *$ & $* * *$ & *** & $* * *$ & FALSE & $* * *$ & $* * *$ & FALSE & FALSE \\
\hline 1983 & $* * *$ & FALSE & $* * *$ & $* * *$ & $* * *$ & *** & FALSE & $* * *$ & FALSE & FALSE \\
\hline 1984 & $* * *$ & $* * *$ & $* * *$ & $* * *$ & $* * *$ & $* * *$ & $* * *$ & $* * *$ & $* * *$ & FALSE \\
\hline 1985 & $* * *$ & FALSE & $* * *$ & $* * *$ & $* * *$ & $* * *$ & $* * *$ & $* * *$ & FALSE & FALSE \\
\hline 1986 & $* * *$ & $* * *$ & $* * *$ & $* * *$ & $* * *$ & $* * *$ & $* * *$ & $* * *$ & $* * *$ & $* * *$ \\
\hline 1987 & $* * *$ & $* * *$ & $* * *$ & $* * *$ & $* * *$ & $* * *$ & $* * *$ & $* * *$ & $* * *$ & FALSE \\
\hline 1988 & $* * *$ & $* * *$ & $* * *$ & FALSE & FALSE & $* * *$ & $* * *$ & FALSE & FALSE & FALSE \\
\hline 1989 & FALSE & $* * *$ & $* * *$ & $* * *$ & $* * *$ & $* * *$ & $* * *$ & $* * *$ & FALSE & FALSE \\
\hline 1990 & $* * *$ & $* * *$ & $* * *$ & $* * *$ & $* * *$ & FALSE & FALSE & FALSE & FALSE & $* * *$ \\
\hline 1991 & $* * *$ & $* * *$ & $* * *$ & $* * *$ & $* * *$ & $* * *$ & FALSE & $* * *$ & FALSE & FALSE \\
\hline 1992 & $* * *$ & FALSE & FALSE & $* * *$ & FALSE & FALSE & FALSE & FALSE & FALSE & $* * *$ \\
\hline 1993 & FALSE & $* * *$ & FALSE & FALSE & FALSE & FALSE & FALSE & FALSE & FALSE & FALSE \\
\hline 1994 & FALSE & FALSE & FALSE & FALSE & FALSE & FALSE & FALSE & FALSE & FALSE & FALSE \\
\hline 1995 & $* * *$ & $* * *$ & FALSE & $* * *$ & FALSE & $* * *$ & FALSE & FALSE & FALSE & FALSE \\
\hline 1996 & $* * *$ & FALSE & $* * *$ & FALSE & FALSE & FALSE & FALSE & FALSE & FALSE & FALSE \\
\hline 1997 & FALSE & $* * *$ & FALSE & FALSE & FALSE & FALSE & FALSE & FALSE & FALSE & FALSE \\
\hline 1998 & $* * *$ & $* * *$ & $* * *$ & FALSE & FALSE & FALSE & FALSE & FALSE & $* * *$ & FALSE \\
\hline 1999 & FALSE & FALSE & $* * *$ & FALSE & FALSE & FALSE & FALSE & FALSE & FALSE & FALSE \\
\hline 2000 & FALSE & $* * *$ & FALSE & FALSE & $* * *$ & $* * *$ & $* * *$ & $* * *$ & FALSE & $* * *$ \\
\hline 2001 & FALSE & FALSE & $* * *$ & FALSE & FALSE & $* * *$ & FALSE & FALSE & FALSE & FALSE \\
\hline 2002 & FALSE & FALSE & FALSE & $* * *$ & FALSE & FALSE & FALSE & FALSE & FALSE & FALSE \\
\hline 2003 & FALSE & FALSE & FALSE & FALSE & FALSE & FALSE & FALSE & FALSE & FALSE & FALSE \\
\hline 2004 & FALSE & $* * *$ & FALSE & FALSE & FALSE & FALSE & FALSE & FALSE & FALSE & FALSE \\
\hline 2005 & $* * *$ & FALSE & FALSE & FALSE & FALSE & FALSE & FALSE & FALSE & FALSE & FALSE \\
\hline 2006 & $* * *$ & $* * *$ & FALSE & FALSE & FALSE & FALSE & FALSE & FALSE & FALSE & FALSE \\
\hline 2007 & $* * *$ & FALSE & FALSE & FALSE & FALSE & FALSE & FALSE & FALSE & FALSE & FALSE \\
\hline
\end{tabular}

(Note: "False" indicates that the Monday returns are not significantly different from zero using the ten percent level of significance using the Wald Test, *** indicates the Monday returns are significantly different from zero at the ten percent level using the Wald Test ) 
Table 5: Wald Tests Results Annual Excess Mean Monday Returns by Year and Capitalization Decile for NASDAQ Stocks

\begin{tabular}{|c|c|c|c|c|c|c|c|c|c|c|}
\hline Year & Decile 1 & Decile 2 & Decile 3 & Decile 4 & Decile 5 & Decile 6 & Decile 7 & Decile 8 & Decile 9 & Decile10 \\
\hline 1973 & $* * *$ & $* * *$ & $* * *$ & $* * *$ & $* * *$ & $* * *$ & $* * *$ & $* * *$ & $* * *$ & $* * *$ \\
\hline 1974 & $* * *$ & $* * *$ & $* * *$ & $* * *$ & FALSE & $* * *$ & $* * *$ & $* * *$ & $* * *$ & $* * *$ \\
\hline 1975 & FALSE & FALSE & FALSE & FALSE & FALSE & $* * *$ & FALSE & FALSE & FALSE & FALSE \\
\hline 1976 & $* * *$ & $* * *$ & $* * *$ & $* * *$ & $* * *$ & $* * *$ & $* * *$ & FALSE & FALSE & FALSE \\
\hline 1977 & $* * *$ & FALSE & FALSE & FALSE & $* * *$ & $* * *$ & $* * *$ & FALSE & $* * *$ & FALSE \\
\hline 1978 & $* * *$ & $* * *$ & $* * *$ & $* * *$ & $* * *$ & $* * *$ & $* * *$ & $* * *$ & $* * *$ & $* * *$ \\
\hline 1979 & FALSE & $* * *$ & FALSE & FALSE & $* * *$ & $* * *$ & $* * *$ & FALSE & $* * *$ & $* * *$ \\
\hline 1980 & $* * *$ & $* * *$ & $* * *$ & $* * *$ & FALSE & $* * *$ & $* * *$ & $* * *$ & *** & $* * *$ \\
\hline 1981 & $* * *$ & $* * *$ & $* * *$ & $* * *$ & $* * *$ & $* * *$ & $* * *$ & $* * *$ & $* * *$ & $* * *$ \\
\hline 1982 & $* * *$ & $* * *$ & $* * *$ & $* * *$ & $* * *$ & $* * *$ & $* * *$ & $* * *$ & $* * *$ & $* * *$ \\
\hline 1983 & $* * *$ & $* * *$ & $* * *$ & $* * *$ & $* * *$ & $* * *$ & $* * *$ & $* * *$ & $* * *$ & $* * *$ \\
\hline 1984 & $* * *$ & $* * *$ & $* * *$ & $* * *$ & $* * *$ & $* * *$ & $* * *$ & $* * *$ & $* * *$ & $* * *$ \\
\hline 1985 & FALSE & $* * *$ & $* * *$ & $* * *$ & $* * *$ & $* * *$ & $* * *$ & $* * *$ & $* * *$ & $* * *$ \\
\hline 1986 & $* * *$ & $* * *$ & $* * *$ & $* * *$ & $* * *$ & $* * *$ & $* * *$ & $* * *$ & $* * *$ & $* * *$ \\
\hline 1987 & $* * *$ & $* * *$ & $* * *$ & $* * *$ & $* * *$ & $* * *$ & $* * *$ & $* * *$ & $* * *$ & $* * *$ \\
\hline 1988 & FALSE & $* * *$ & $* * *$ & $* * *$ & $* * *$ & $* * *$ & $* * *$ & $* * *$ & $* * *$ & FALSE \\
\hline 1989 & $* * *$ & $* * *$ & $* * *$ & $* * *$ & $* * *$ & $* * *$ & $* * *$ & $* * *$ & $* * *$ & $* * *$ \\
\hline 1990 & FALSE & $* * *$ & $* * *$ & $* * *$ & $* * *$ & $* * *$ & $* * *$ & $* * *$ & FALSE & FALSE \\
\hline 1991 & $* * *$ & $* * *$ & $* * *$ & $* * *$ & $* * *$ & $* * *$ & $* * *$ & $* * *$ & $* * *$ & FALSE \\
\hline 1992 & FALSE & $* * *$ & $* * *$ & $* * *$ & $* * *$ & $* * *$ & $* * *$ & FALSE & FALSE & FALSE \\
\hline 1993 & $* * *$ & $* * *$ & $* * *$ & $* * *$ & $* * *$ & $* * *$ & $* * *$ & $* * *$ & FALSE & FALSE \\
\hline 1994 & $* * *$ & $* * *$ & $* * *$ & $* * *$ & FALSE & $* * *$ & $* * *$ & $* * *$ & FALSE & FALSE \\
\hline 1995 & $* * *$ & FALSE & $* * *$ & $* * *$ & $* * *$ & $* * *$ & FALSE & FALSE & $* * *$ & FALSE \\
\hline 1996 & $* * *$ & $* * *$ & $* * *$ & FALSE & $* * *$ & FALSE & FALSE & FALSE & FALSE & FALSE \\
\hline 1997 & $* * *$ & $* * *$ & $* * *$ & FALSE & FALSE & FALSE & FALSE & FALSE & FALSE & FALSE \\
\hline 1998 & $* * *$ & $* * *$ & $* * *$ & $* * *$ & $* * *$ & $* * *$ & $* * *$ & FALSE & FALSE & FALSE \\
\hline 1999 & $* * *$ & $* * *$ & FALSE & FALSE & FALSE & FALSE & FALSE & FALSE & FALSE & $* * *$ \\
\hline 2000 & FALSE & $* * *$ & FALSE & FALSE & FALSE & FALSE & FALSE & FALSE & FALSE & FALSE \\
\hline 2001 & $* * *$ & FALSE & FALSE & FALSE & FALSE & FALSE & FALSE & FALSE & FALSE & FALSE \\
\hline 2002 & $* * *$ & $* * *$ & $* * *$ & $* * *$ & $* * *$ & $* * *$ & FALSE & FALSE & FALSE & FALSE \\
\hline 2003 & FALSE & FALSE & FALSE & FALSE & FALSE & FALSE & FALSE & FALSE & FALSE & FALSE \\
\hline 2004 & FALSE & FALSE & $* * *$ & $* * *$ & FALSE & FALSE & FALSE & FALSE & FALSE & FALSE \\
\hline 2005 & FALSE & FALSE & FALSE & FALSE & FALSE & FALSE & FALSE & FALSE & FALSE & FALSE \\
\hline 2006 & FALSE & FALSE & FALSE & $* * *$ & $* * *$ & FALSE & FALSE & FALSE & FALSE & FALSE \\
\hline 2007 & FALSE & FALSE & FALSE & FALSE & FALSE & FALSE & FALSE & FALSE & FALSE & FALSE \\
\hline
\end{tabular}

(Note: "False" indicates that the Monday returns are not significantly different from zero using the ten percent level of significance for the Wald Test, *** indicates the Monday returns are significantly different from zero at the ten percent level using the Wald Test ) 